\title{
Concerning Magnetic Field Measurements in Prominences Using Zeeman and Hanle Methods
}

\author{
V.S. Bashkirtsev and G.P. Mashnich \\ Institute of Solar-Terrestrial Physics, Irkutsk, P.O. Box 4026, Russia
}

\begin{abstract}
A critical review is made of the results of magnetic field measurements in prominences obtained by the Zeeman and Hanle methods. By considering the example of three prominences, some discrepancies between the methods are shown. The authors consider that the magnetic field measurements by the Hanle method inadequately represent the actual magnetic field pattern. To overcome these discrepancies a comparative analysis of magnetic field measurements in prominences by both methods is required.
\end{abstract}

\section{Introduction}

Prominence magnetic fields are currently measured using the Zeeman and Hanle effects. While, however, the Zeeman method was put through adequate tests in its practical implementation for magnetic field measurements on the Sun and the results obtained are beyond question, the Hanle method has not been validated at all. Sahal-Brechot et al. (1977) did make estimates only, based on which they arrived at the conclusion that the effects of emission depolarization and of rotation of the vector of polarization observed in prominences are caused by the magnetic field alone. It should immediately be noted that diagnosing the magnetic field on the basis of the Hanle effect is built precisely on this conclusion. However, our subsequent short comparative analysis of observational data (Figures 1-3) obtained by both methods runs counter to this estimating conclusion. We now address the observational data.

\section{Observations and Discussion}

Results of polarimetric measurements (Hanle method) in a clearly defined giant arch-like prominence (Figure 1) observed on August 15-17, 1980 at the latitude $\varphi=-25^{\circ} \mathrm{E}$ at Sacramento Peak Observatory (Athay et al. 1983) are surprising: the magnetic field vector runs parallel to the solar surface throughout the entire arch-like prominence, traverses its body across the arch (at the angle of $86^{\circ}$ ) and is directed from the positive polarity of the photospheric magnetic field to the negative polarity. The height gradient of the magnetic field was not determined. In our magnetograph observations on August 15-17 (Zeeman method), we recorded a positive height gradient of magnetic field $B_{\|}$, an increase of $B_{\|}$ toward the arch top (Bashkirtsev and Mashnich 1987). This disagrees with data of polarimetric measurements but logically follows from the picture that the 


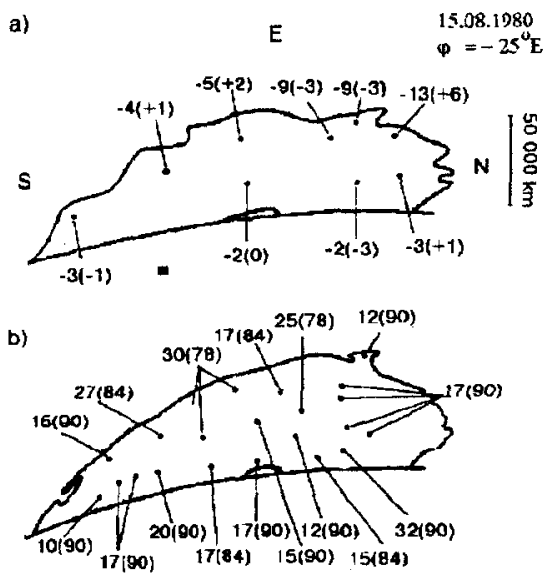

Figure 1. Sketches of the prominence on August 15, $1980\left(\varphi=-25^{\circ}\right.$ E). a) Magnetograph measurements at the Sayan Solar Observatory: first numerals are the measured longitudinal magnetic field strengths $B_{\|}$in $\mathrm{G}$ (minus = negative polarity); numerals in brackets = line of sight velocities in $\mathrm{km} \mathrm{s}^{-1}$ (plus and minus signs denote, respectively, the direction from and to observer). b) Polarimetric measurements at Sacramento Peak Observatory: first numerals = the magnetic field strengths (modulus of B); numerals in brackets = the deviation of the magnetic field vector from the local vertical to the solar surface (in degrees).

magnetic field is directed along the prominence arch.

Another prominence, for which it is also possible to compare measurements obtained by both methods, was observed on August 5, 1980 at the latitude $\varphi=+22^{\circ} \mathrm{E}$ (Figure 2). It is remarkable that the emission features of this prominence were arranged along a path resembling a loop. From an interpretation of polarimetry data (Querfeld et al. 1985) it follows that, with increasing height, the magnetic field remains horizontal, i.e., parallel to the solar surface, quasiperpendicular to the neutral line of the photospheric magnetic field and has a normal polarity. At the same time, according to our observations (Bashkirtsev and Mashnich 1987), both the longitudinal component of the magnetic field and the line-of-sight velocity of mass motion in the prominence increases with the height. Thus, we find that the magnetic field clearly changes with height, which cannot be said based on polarimetric measurements. The following comparison of magnetograph measurements with polarimetry data (courtesy J.L. Leroy) can be made for the prominence $\varphi=+75^{\circ} \mathrm{E}$ (Figure 3) observed on August 13, 1980 (Bashkirtsev and Mashnich 1987). The presence of two polarities in this prominence according to our observations indicates that the magnetic field has a more complicated configuration compared with the interpretation of polarimetric measurements: the magnetic field is unipolar, the magnetic field vector is parallel to the solar surface and quasi-perpendicular to the prominence long axis. On the whole, analysis of the polarized emission in terms of the Hanle 


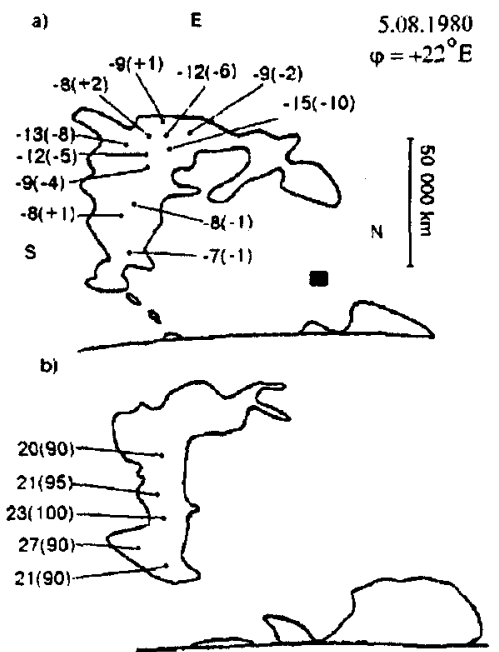

Figure 2. Sketches of the prominence on August 5, $1980\left(\varphi=+22^{\circ}\right.$ E). a) Magnetograph measurements at the Sayan Solar Observatory. b) Polarimetric measurements at Sacramento Peak Observatory. For the other designations see Figure 1.

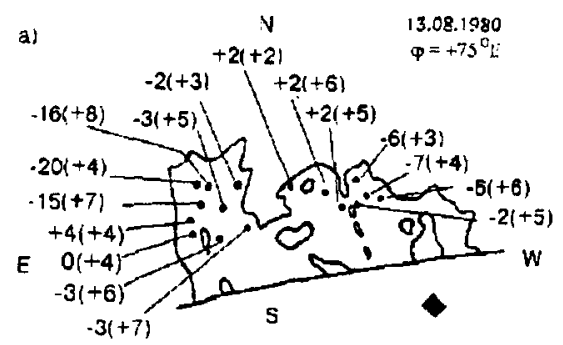

b)

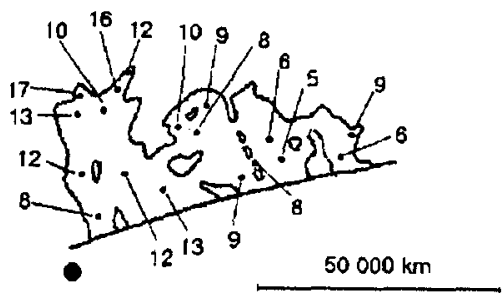

Figure 3. Sketches of the prominence on August 13, $1980\left(\varphi=+75^{\circ}\right.$ E). a) Magnetograph measurements at the Sayan Solar Observatory. Dark box $=$ spectrograph aperture. For other designations see Figure 2(a). b) Polarimetric measurements at the Pic du Midi Observatory. Numerals = magnetic field strength (modulus of B) in G. Dark circle $=$ spectrograph aperture. 


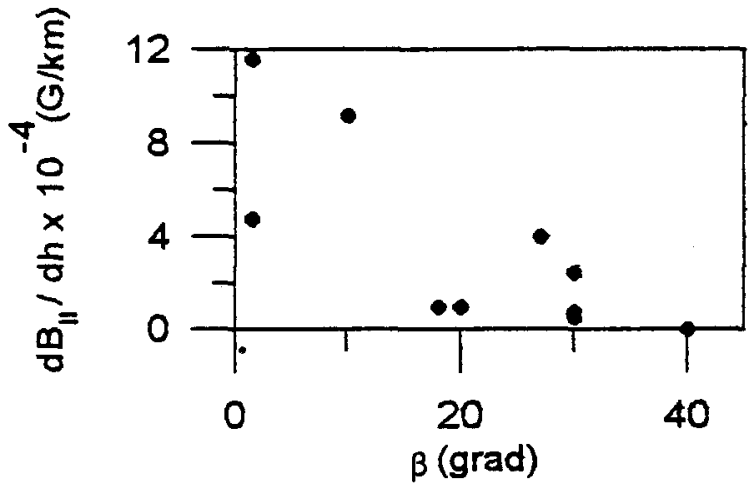

Figure 4. Dependence of the height gradient of the longitudinal magnetic field, $d B_{\|} / d h$, in prominences on the $\beta$ angle between the line of sight and the prominence (filament) long axis.

effect shows that the magnetic field in quiescent prominences is always invariably horizontal: the deviation from the horizontal (solar surface) does usually not exceed $10-15^{\circ}$. Our data are in conflict with this inference, however. The non-horizontal arch-like magnetic field is favoured by the following fact. If the magnetic field has a arch-like character, then the height gradient of the longitudinal field, $d B_{\|} / d h$, must depend on the angle $\beta$ between the line of sight and the filament long axis and must increase with decreasing $\beta$. Such a dependence (taken from Bashkirtsev and Mashnich (1987)) ' is presented in Figure 4, and one can see that the expected behaviour of the height gradient $B_{\|}$is indeed observed. Hence, the view of a arch-like spiral prominence magnetic field is supported by Zeeman observations, as well as by other observational evidence which, unfortunately, cannot be included here because of lack of space, but such a view is inconsistent with results obtained using the Hanle effect. To make sure that the Hanle method is valid when applied to prominences, it is necessary to carry out a further detailed comparison of observational data obtained by both methods.

Acknowledgments. This work was supported by the Russian Foundation for Fundamental Research under grant 960216647 and 970226726 , and Governmental Support Grant for Leading Scientific Schools 961596733.

\section{References}

Athay, R.G., Querfeld, C.W., Smartt, R.N., Landi Degl' Innocenti, E. and Bommier, V. 1983, Solar Phys., 89, 3

Bashkirtsev, V.S. \& Mashnich, G.P.. 1987, in The Magnetographic Observations of Prominences at the Sayan Observatory, Irkutsk: preprint, 1987, p. 52

Querfeld, C.W., Smartt, R.N., Bommier,V., Landi Degl' Innocenti, E. and House, L.L 1985, Solar Phys., 96, 277

Sahal-Brechot, S., Bommier, V. and Leroy, J.L. 1977, A\&A., 59, 223 